\title{
Zwischen Anspruch und Wirklichkeit: Patientenkompetenz und Leitlinien-orientierte Medizin - ein Plädoyer
}

\section{Zusammenfassung}

Leitlinien von Fachgesellschaften sind häufig Grundlage für Therapieentscheidungen. Wenn die Leitlinienempfehlungen nicht als Empfehlungen, sondern als Teil eines medizinisch unbestrittenen und dadurch notwendigen Behandlungskatalogs wahrgenommen werden, sind Konflikte zwischen Arzt und mündigem Patienten vorprogrammiert. Dieser Beitrag ist ein Plädoyer für eine menschliche und wirklich integrative Medizin am Beispiel der Brustkrebstherapie, indem mit der Patientin gemeinsam eine Entscheidung gefunden wird, auch wenn dies im Einzelfall bedeutet, dass Leitlinienempfehlungen aus individuellen Erwägungen heraus abgelehnt werden.

Schlüsselwörter: Patientenkompetenz, Leitlinien, Mammakarzinom, onkologische Therapie, antihormonelle Therapie, Chemotherapie, Bestrahlung, Lymphonodektomie

\section{Brauchen wir eine neue Kultur der Integration?}

Diese Frage möchte ich in Bezug auf die Vereinbarung von Patientenkompetenz und Leitlinienempfehlungen stellen. Patientenkompetenz und Leitlinien schließen sich nicht von vornherein aus, können sich aber erheblich behindern, wenn die Leitlinienempfehlungen nicht als Empfehlungen, sondern als Teil eines medizinisch unbestrittenen und dadurch notwendigen Behandlungskatalogs wahrgenommen werden. Leitlinien sind laut Prof. emer. Peter Matthiessen von der Universität Witten/ Herdecke „auf allgemeine Krankheitseinheiten und -situationen bezogen; der Behandlungsauftrag des Arztes richtet sich aber auf konkrete und damit unaustauschbare Kranke“ [22].

Nicht zuletzt zeigt sich der Widerspruch vor allem in dem wachsenden Patientenbedürfnis nach zunehmender Selbstbestimmung und Mündigkeit. So wünschen sich 2/3 der Patientinnen mit neu diagnostiziertem Brustkrebs eine akti- ve Einbeziehung in die Therapieentscheidungen [8, 31, 32].

Mit dem neuen Begriff der „individualisierten“ oder auch „personalisierten“ Medizin verbindet sich die Erwartung, dass medizinische Therapien für den Patienten maßgeschneidert werden. Allerdings ergeben sich erhebliche Differenzen, betrachtet man die Ansprüche, die aus Patientensicht und dem Verständnis einer mechanistischen Medizin resultieren. Die Problematik wird dadurch noch verschärft, dass das Entscheidungsspektrum stetig durch neue Entwicklungen in Diagnostik und Therapie erweitert und selbst für den Experten stellenweise unübersichtlich wird.

Eine am Patienten orientierte ,individualisierte“ Therapieplanung beinhaltet neben der Kenntnis der Leitlinien auch die Interpretation der Leitlinien im Zusammenhang mit den Lebensumständen des Patienten. Voraussetzung dafür ist eine Vertrauensbasis zu einer begrenzten Zahl von Ärzten, die den Patienten in seiner Ganzheit sehen, annehmen, beraten
Nicole Weis

und begleiten. Bisher fehlt es dabei an den dafür geeigneten Rahmenbedingungen, der Zeit für die sondierenden Gespräche des Patienten mit den in die Behandlung involvierten Ärzten sowie auch häufig der Zeit für eine ausreichende Kommunikation der betreuenden Ärzte untereinander. Hinzu kommt noch, dass das ärztliche Gespräch keine adäquate Anerkennung im Honorarsystem findet [16].

\section{Informationsbedarf der Patienten}

Dass für 70\% der Krebspatienten das persönliche Gespräch mit dem Arzt die wichtigste Informationsquelle ist, bestätigt eine aktuelle Studie aus der Universität Frankfurt/Main [29]. Bei Angehörigen ist dies anders: Knapp die Hälfte der Befragten wendet sich an Selbsthilfegruppen, knapp 30\% suchen das direkte Gespräch in Krebsberatungsstellen, und fast die Hälfte der befragten Angehörigen von Krebspatienten informiert sich im Internet. Bei der Wahl der Informationsquelle standen die individuelle und umfassende Information, hohes Expertenwissen, die Möglichkeit, Fragen zu stellen und ein konstanter Ansprechpartner im Vordergrund.

Diese Studie bestätigt die praktische Erfahrung in der Beratung an Krebs erkrankter Menschen, dass die Patienten eine individuelle Beratung suchen, die sie bei ihrem persönlichen Umgang mit der Krankheit Krebs unterstützt (Abb.1). Die Erfahrungen der Gesellschaft für Biologische Krebsabwehr (GfBK) seit über 33 Jahren zeigen auch, dass besonders Brustkrebspatientinnen den Wunsch haben, komplementärmedizinisch beraten zu werden. 
Hierbei spielt neben der fachlichen Kompetenz auch der Faktor Zeit eine wichtige Rolle. Demgegenüber steht der Zeitmangel in Klinik und Praxis, die der Krebspatient dann erfährt, wenn er mit seiner Erkrankung einen Arzt aufsucht und individuelle Fragen sowie kritische Rückfragen stellen möchte. Spätestens dann wird der Patient damit konfrontiert, dass der Faktor Zeit in der Alltagsroutine von Kliniken und onkologischen Praxen Mangelware ist. So dauert das durchschnittliche ärztliche Gespräch laut dem Arztreport der Barmer GEK 2010 gerade mal 8 Minuten.

Verschärft wird die enttäuschende Erfahrung noch durch die weit verbreitete Praxis, dass im ohnehin zu kurzen Arztgespräch Zeitdruck und Angst aufgebaut werden und der Patient dazu gedrängt wird, die Operation oder Chemotherapie innerhalb kürzester Zeit durchführen zu lassen. Dabei ist Brustkrebs kein Notfall! So zeigte sich in einer dänischen Studie mit 750 Frauen, dass ein früher Beginn einer Chemotherapie innerhalb der ersten drei Wochen nach Operation keinen Überlebensvorteil bedeutet [5].

\section{Heilfaktor Arzt}

Schon Paracelsus erkannte im 16. Jahrhundert: „Die Kraft des Arztes liegt im Patienten.“ Dies bedeutet, dass es im Arztgespräch auch wichtig ist, das Heilsame im Patienten zu stärken. Wenn der Arzt das Vertrauen des Patienten gewinnt, dann begünstigt dies wiederum Faktoren, die sich positiv auf die medizinische Intervention auswirken [27].

Wie bedeutsam das Gespräch mit dem Arzt ist, bestätigen Studien, in denen empathisches ärztliches Kommunikationsverhalten mit einer besseren psychischen Anpassung bei Brustkrebs assoziiert war [28]. Unabhängig vom Gesprächsinhalt führt eine schlechte Kommunikation zu andauernder Belastung und Verunsicherung aufseiten der Patienten, während eine gute ärztliche Kommunikation als hilfreich bei der Bewältigung und Akzeptanz einer Krebserkrankung empfunden wird [20]. Außerdem führt die stärkere Einbindung der Patienten in Entscheidungen zu einer

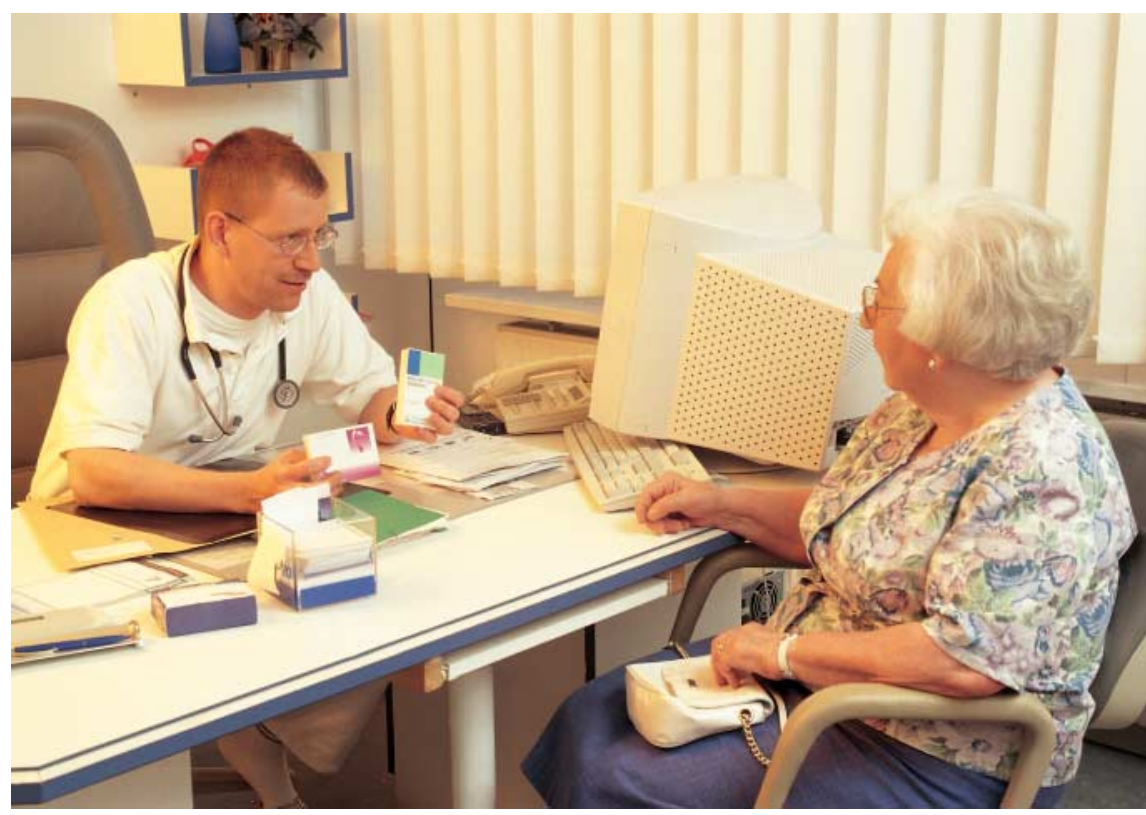

Abb. 1: Patienten suchen eine individuelle ärztliche Beratung, die von Vertauen und Empathie getragen ist. Foto: MEV

höheren Zufriedenheit der Patienten mit dem Arzt [21].

Die Erfahrung und Rückmeldung von Patienten zeigt jedoch, dass auf kritische Nachfragen von Patienten unterschiedlich und leider oft mit Unverständnis reagiert wird. Dabei liegt nichts näher als die positiven Aspekte von wachsender Patientenkompetenz hervorzuheben. So erklärt der Onkologe Prof. Gerd Nagel, der ehemalige Präsident der Deutschen Krebsgesellschaft: „Viele Ärzte verstehen einfach nach wie vor nicht, dass sich kompetente Patienten gar nicht in die Angelegenheit der Ärzte, sondern in die eigenen Belange einmischen wollen.“ (Weitere Infomationen unter www.patientenkompetenz.ch.)

\section{Problem der Übertherapie}

An dieser Stelle möchte ich die Problematik einer Übertherapie beim Mammakarzinom hervorheben, der in den Leitlinien bisher nur unvollständig begegnet wird. Dabei ist eine zentrale Frage, ob Patientinnen, denen bei einem geringen absoluten Rückfallrisiko durch eine adjuvante Chemotherapie nicht nur nicht geholfen, sondern durch die Toxizität dieser Chemotherapie eher geschadet wird. Dass bei aller Euphorie über die gestiegene Lebenserwartung bei Brustkrebserkrankten nicht die langfristigen Nebenwirkungen übersehen werden dürfen, forderte Prof. Christoph Thomssen aus Halle auf dem Symposium „Brustkrebs überlebt - und dann? Langzeitfolgen bei jungen Frauen“ anlässlich der 35. Jahrestagung der Deutschen Gesellschaft für Senologie im Juni 2015 in Leipzig.

Beispielhaft zur möglichen Übertherapie durch eine adjuvante Chemotherapie beim Mammakarzinom sei die britische TACT-Studie zitiert [10], in der gezeigt wurde, dass eine taxanhaltige Chemotherapie das Rezidivrisiko und die Sterblichkeit bei Frauen mit Brustkrebs im Frühstadium - verglichen mit einer anthrazyklinbasierten Standardchemotherapie - im Gegensatz zu früheren Studien nicht reduziert. Zudem war in einer Folgestudie bei den Frauen, die eine taxanhaltige Chemotherapie erhielten, im Vergleich zu Patientinnen mit einer anthrazyklinbasierten Standardtherapie eine höhere Toxizität und stärkere Beeinträchtigungen während der Therapie zu beobachten [13].

Unter dem Titel „Taxan bei frühem Brustkrebs: Mehr Schaden als Nutzen?" [25] liest sich dazu ein bemerkenswerter Kommentar von Prof. Michael Untsch aus Berlin-Buch: „Aktuell wissen wir zwar nicht, wie viele Übertherapien wir in den letzten zehn Jahren durchgeführt haben, 


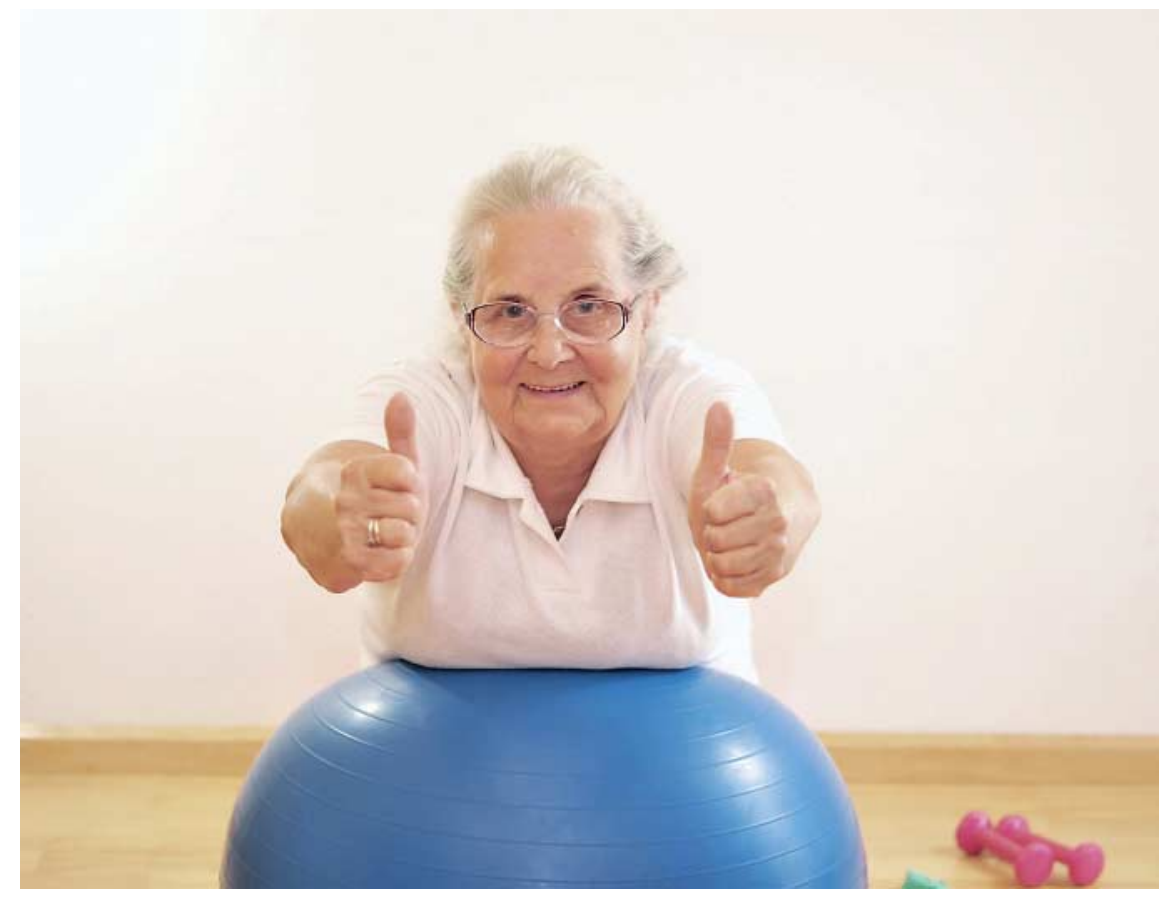

Abb. 2: Moderater Ausdauersport kann die Brustkrebssterblichkeit um rund $50 \%$ senken ohne Nebenwirkungen. Foto: Fotolia/Printemps

aber es dürften nicht wenige gewesen sein. Denn wenn die Angst der Hauptgrund für die Indikation ist, bleiben sachliche Gründe oft auf der Strecke." Weiter heißt es: „Die Studie von Hall und Kollegen soll uns Therapeuten erneut etwas Wichtiges in Erinnerung rufen: Wenn wir im multidisziplinären Tumorboard zusammensitzen, ist die einzelne Patientin keine Zusammensetzung aus Abkürzungen (TNM, Grading, ER, PR, HER 2, Ki 67, Nodalstatus). Sie ist ein Mensch, der zwar mit der Diagnose Krebs konfrontiert ist, aber etwas ganz Wichtiges hat, das es zu erhalten gilt: die Lebensqualität. Ihr müssen wir uns genauso verpflichtet fühlen wie der onkologischen Diagnose.“ [25]

Laut Prof. Untsch bleiben die Taxane trotzdem ein wesentlicher Bestandteil der adjuvanten Chemotherapie. Er begründet dies damit, dass unterm Strich die relative Risikoreduktion für Rezidive und Sterblichkeit etwa $20-30 \%$ beträgt. In der Diskussion um den Nutzen einer adjuvanten Chemotherapie ist aber vor allem eines wichtig zu wissen und einzuschätzen: die genaue Kenntnis der Begriffe „absolute Risikoreduktion“ und „relative Risikoreduktion".
Dazu ein Beispiel: Eine Patientin hat ein Rückfallrisiko von $60 \%$. Eine adjuvante Therapie kann das Rückfallrisiko um relative $33 \%$, also um ein Drittel reduzieren. Da $20 \%$ ein Drittel von $60 \%$ ist, ist die absolute Risikoreduktion $20 \%$ und die relative Risikoreduktion $33 \%$. Also hat die Patientin nach einer adjuvanten Therapie ein Gesamt-RestRisiko von $40 \%$.

Zum Vergleich eine Patientin, deren Rückfallrisiko bei nur 15\% liegt: Deren absolute Risikoreduktion beträgt nur $5 \%$, verspricht jedoch einen relativen Vorteil von 33\% [19]. Das heißt, der Gewinn durch eine Chemotherapie ist bei größerem Rückfallrisiko eher moderat, bei geringem Rückfallrisiko sogar deutlich geringer als die relativen Zahlen vermuten lassen.

Wenn man sich die absoluten Zahlen einer Zusammenfassung von etwa 33.000 Patientinnen aus 102 Studien zum Nutzen einer Chemotherapie anschaut, sind diese ebenfalls eher ernüchternd: So reduzierte bei Frauen unter 50 Jahren eine Chemotherapie das Rückfallrisiko um 12,4\% und das Sterberisiko um 10,0\%. Bei Frauen zwischen 50 und 69 Jahren reduzierte sich das Rückfallrisiko um 4,2\% und das Sterberisiko um 3,0\% [23]. Umso wichtiger ist es, dass das Rezidiv- und Metastasierungsrisiko mit Genanalysen genauer eingeschätzt wird, um die adjuvante Chemotherapie bei Brustkrebs nur den Frauen zu empfehlen, die sie auch wirklich benötigen.

Aber selbst wenn statistisch gesehen die Wahrscheinlichkeit hoch ist, dass eine Chemotherapie Erfolg verspricht, bedeutet das noch lange nicht, dass die verbliebenen Tumorzellen auch tatsächlich auf die Chemotherapie ansprechen. Prof. Hermann Faller, Medizinpsychologe an der Universität Würzburg, bringt die Problematik wie folgt auf den Punkt: „Es gibt keine $100 \%$ ige Sicherheit, sondern nur Wahrscheinlichkeiten.“ Und weiter: „Das darf nicht dazu führen, dass man Sicherheiten vermittelt, die in Wirklichkeit nicht da sind.“ [17]

Nicht zuletzt sollte man bei der ganzen Diskussion um eine adjuvante Chemotherapie oder eine antihormonelle Therapie einen wichtigen Punkt nicht außer Acht lassen: In verschiedenen Studien senkte moderater Ausdauersport, das heißt wöchentlich etwa drei bis fünf Stunden Laufen oder gleichwertige Übungen, das Risiko, an Brustkrebs zu sterben, um relativ bis zu 51\% [2] (Abb.2). Offenbar gibt es auch einen Dosiseffekt: Je mehr körperliche Aktivität, umso bessere Wirksamkeit, besonders bei Frauen nach den Wechseljahren [4, 34]. Der World Cancer Research Fund (WCRF) empfiehlt, täglich mindestens 30 Minuten moderat körperlich aktiv zu sein. Als besonders vorteilhaft hat sich ein kombiniertes Kraft- und Ausdauertraining erwiesen [11].

Frau Prof. Marion Kiechle, Direktorin der Frauenklinik des Klinikums rechts der Isar der Technischen Universität München, sagte zum Thema Bewegung anlässlich des Symposiums „Sport und Krebs“ 2008 in München: „Derartige Resultate, das muss man ganz ehrlich sagen, erreichen wir mit keiner anderen Therapie, sei es Chemooder Antikörpertherapie!“ Und auf der Webseite der Deutschen Krebsgesellschaft steht zum Thema Sport: „Bei Frauen mit hormonabhängig wachsendem Brustkrebs senkt Sport den Östrogenspiegel in Blut 
und Gewebe - ebenso wie eine medikamentöse antihormonelle Therapie.“

Außerdem besteht die Gefahr, wenn Entscheidungen nur aufgrund von Leitlinien getroffen werden, dass andere Grundlagen medizinischen Wissens, wie zum Beispiel die ärztliche Berufserfahrung, nicht berücksichtigt werden. Denn Leitlinien stützen ihre Empfehlungen auf die Ergebnisse von Placebo-kontrollierten Studien, die häufig von Pharmaunternehmen gesponsert werden. Kritiker bemerken zu Recht, dass nicht immer eine Kontrolle über Interessenskonflikte der Ärzte erfolgt, die in Leitlinienkommissionen von Fachgesellschaften sitzen [24].

Problematisch ist auch, dass aktuelle Studienergebnisse nur verzögert Eingang in die Leitlinienempfehlungen finden. So kann es sein, dass, obwohl eindeutige Studienergebnisse vorliegen, diese Therapiemöglichkeiten aber noch nicht empfohlen werden. Ein Beispiel ist die brusterhaltende Operation. Bis sich diese Operationsmethode als Standard durchsetzte, waren sechs aufwendige Studien nötig, obwohl bereits eine qualitativ hochwertige Studie deren Vorteile eindeutig nachgewiesen hatte.

\section{Weniger kann mehr sein}

Nachfolgend einige Beispiele einer Übertherapie beim Mammakarzinom, die jedoch in der Praxis kaum zu konkreten Negativempfehlungen geführt haben.

\section{Strahlentherapie bei über 70-Jährigen}

Eine Strahlentherapie der Brust ist nicht nebenwirkungsarm, da sie nicht nur zu einer akuten Rötung der Brust, sondern auch zu Spätfolgen wie Ödemen, Hautveränderungen, Lungenfibrosen und Herzschäden führen kann. Obwohl in frühen Tumorstadien bei älteren Frauen die zusätzliche Strahlentherapie der Brust wenig zu nützen scheint, werden trotzdem immer noch knapp zwei Drittel aller über 70-Jährigen mit Mammakarzinom im Frühstadium bestrahlt (Abb.3). Und dies, obwohl nicht nur die CALGB-9343-Studie aus dem Jahr 2004, sondern auch die Daten der PRIME-II-Studie [18] zeigen konn-

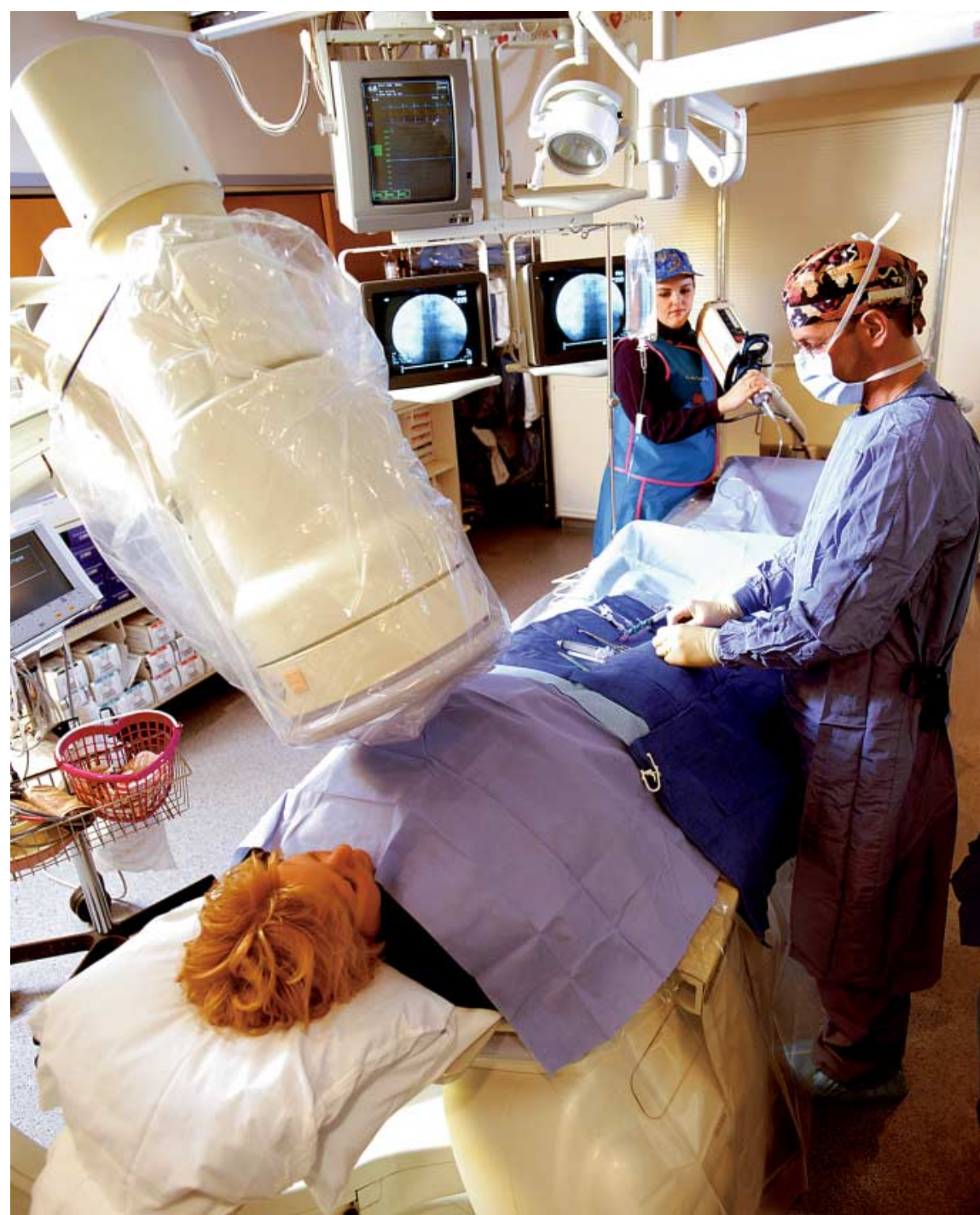

Abb. 3: Der Nutzen einer Strahlentherapie im frühen Brustkrebsstadium bei älteren Patientinnen ist fragwürdig. Foto: Dynamic Graphics

ten, dass der Nutzen bei älteren Frauen mehr als fragwürdig ist.

Bereits im Jahr 2005 wurden auf der Grundlage der Erkenntnisse aus der CALGB-9343-Studie die „National Comprehensive Cancer Network Guidelines“ der USA (vergleichbar mit den Leitlinien der Deutschen Fachgesellschaften) mit folgender Fußnote geändert: „Eine Bestrahlung der Brust kann bei über 70-jährigen Frauen entfallen, die einen Östrogenrezeptor-positiven, nodal-negativen T1-Tumor haben und die eine adjuvante Hormontherapie erhalten.“

Anhand von Daten des US-amerikanischen SEER-Registers (amerikanisches Krebsregister) wurde nun geprüft, ob solche Erkenntnisse das Therapieverhalten in der Praxis verändert haben [26]. Dies war leider nicht der Fall. Es zeigte sich, dass in den Jahren vor Veröffentlichung der CALGB-9343-Studie der Anteil von älteren Mammakarzinompatientinnen und einer Radiotherapie bei etwa 70\% lag. Zwischen 2003 und 2005 sank er auf etwa 60\% und stieg dann wieder leicht auf $62 \%$ an. Der Unterschied bei der Behandlung vor und nach 2004 war zwar signifikant, aber nicht sehr ausgeprägt.

\section{Behandlung des DCIS}

Grundsätzlich ist ein DCIS (ductales carcinoma in situ = Krebsvorstufe der Brustdrüse) aufgrund der guten Prognose auch ohne invasive Behandlungsmethoden the- 
rapierbar. Vor wenigen Jahren wurde noch empfohlen, bei einer Tumorgröße kleiner als $2 \mathrm{~cm}$ (G1 und $1 \mathrm{~cm}$ Sicherheitsabstand) auf eine Bestrahlung zu verzichten. Aktuellen Studien zufolge ist das Rückfallrisiko ohne Bestrahlung nach 25 Jahren um 50\% erhöht, weshalb seit einigen Jahren eine Strahlentherapie empfohlen wird.

Andererseits zeigen die absoluten Zahlen nach der großen UK/ANZ-DCIS-Studie nach 12,7 Jahren nur eine Reduktion der Rückfallrate von $19,4 \%$ auf $7,1 \%$, also um $12,3 \%$-Punkte [6]; in der SweDCIS-Studie waren es nach 20 Jahren kumulative $10 \%$ [33]. In einer europäischen Studie zeigte sich neben einer Reduktion der Rückfallrate um $11 \%$, dass vor allem Frauen unter 40 Jahren mit mäßig oder schlecht differenzierten Tumoren und nicht eindeutig tumorfreien Operationsrändern von einer Strahlentherapie bei DCIS profitierten [3].

Bei diesen Zahlen muss die Frage erlaubt sein, ob bei DCIS nicht auch eine Teilbestrahlung der Brust ausreichend ist [15], zumal eine Verbesserung des Gesamtüberlebens durch eine Strahlentherapie nicht erreicht wird. Immerhin zeigt die UK/ANZDCIS-Studie auch, dass lokale Rückfälle nur durch Bestrahlung (und nicht durch Tamoxifen) verhindert werden.

\section{Antihormonelle Therapie über 10 Jahre}

In der Regel wird eine antihormonelle Therapie über fünf Jahre empfohlen. Der Trend, bei Frauen, die nach fünf Jahren Tamoxifen-Therapie in die Wechseljahre kommen, die antihormonelle Therapie um weitere fünf Jahre mit einem Aromatasehemmer oder mit Tamoxifen zu verlängern, ist umstritten. So sank die Gesamtzahl der Rezidive von 25,1\% auf 21,4\% (also um 3,7\%-Punkte) und die Brustkrebssterberate von $15,0 \%$ auf $12,2 \%$ (also um 2,8\%-Punkte), wenn Tamoxifen über 10 Jahre gegeben wurde [7]. Den geringen Vorteilen stehen Nebenwirkungen und Langzeitfolgen gegenüber. So wurde Gebärmutterkrebs in den Jahren 5 bis 14 bei 3,1\% der Frauen mit 10-jähriger Tamoxifentherapie und bei 1,6\% der Frauen mit 5-jähriger Tamoxifentherapie diagnostiziert. Außerdem kam es zu einem häufigeren Auftreten von Lungenembolien.
Von den Experten der St. Gallener Brustkrebskonferenz in Wien 2015 wird die erweiterte antihormonelle Therapie vor allem bei einem Befall von mehr als 4 Lymphknoten oder G3-Tumoren empfohlen. Vermutlich profitieren nur wenige Patientinnen von dieser erweiterten Therapiestrategie.

\section{Axilläre Lymphknotenentfernung}

Im Jahr 2011 zeigte eine große Studie an 891 Frauen mit Mammakarzinom [12], dass Betroffene mit günstigem Risikoprofil keine Vorteile durch die zusätzlich durchgeführte Lymphknotenentfernung haben, auch wenn der Wächterlymphknoten befallen war, da sich die Heilungschancen dadurch nicht verbesserten. So empfehlen die Autoren dieser Studie, auf eine Lymphknotenentfernung bei Frauen zu verzichten, die brusterhaltend operiert, mit einer Strahlentherapie nachbehandelt werden und die folgende Tumorkonstellation aufweisen: cT1-2, cN0, G1-2, 1-2 befallene Wächterlymphknoten. Bisher stellen die S3-Leitlinien zu diesem Thema vorsichtig fest, dass „bei Patientinnen mit postitivem Sentinel-Lymphknoten ein Verzicht auf jegliche axilläre Intervention in Ausnahmesituationen erwogen werden kann“.

Eine wegweisende Studie zur Frage, ob wirklich alle Lymphknoten entfernt werden müssen, startete 2015 mit der deutschen INSEMA-Studie (Intergroup-Sentinel-Mamma), in der am krankheitsfreien Überleben überprüft wird, ob bei einem bis zu fünf Zentimeter großen Mammakarzinom bei unauffälligem Befund der Axilla und geplanter brusterhaltender Therapie auf die Entfernung des Wächterlymphknotens verzichtet werden kann. Beteiligt sein werden fast 6000 Patientinnen mit frühem Brustkrebs aus Deutschland an etwa 130 Studienzentren sowie etwa 800 Patientinnen in Österreich [14].

\section{Weitere Beispiele und Herausforderun- gen der Zukunft}

Weitere Beispiele einer Übertherapie, bei denen in Studien nur geringe Effekte auftraten, sind die Ovar-Ablation bei nach der Chemotherapie wieder einsetzender
Menstruation sowie die Strahlentherapie nach Ablatio der Brust. So empfehlen letzteres die Leitlinien deutscher Fachgesellschaften nur bei weit ausgedehnten Tumoren, bei verbliebenem Tumorrest und bei mehr als drei befallenen Achsellymphknoten. Dass die Postmastektomiebestrahlung in der letzten Zeit zunehmend auch bei 1-3 befallenen Lymphknoten empfohlen wird, geht auf eine Studie zurück, deren Aussagekraft noch diskutiert wird, da in dieser Metaanalyse Daten zwischen 1964 und 1986 ausgewertet wurden [8] und man hier zu Recht fragen kann, ob sich die relative Risikoreduktion auch auf aktuelle Patientinnen übertragen lässt.

In Zukunft wird die Herausforderung einer individualisierten Brustkrebstherapie vor allem darin liegen, die Patienten herauszufiltern, die von einer leitliniengerechten Therapie profitieren und diese von der Mehrzahl der Patienten zu unterscheiden, bei denen weniger Therapie den gleichen Nutzen bedeutet.

Aktuell wird vor allem bei der adjuvanten Chemotherapie mit sogenannten genomischen Testverfahren versucht, die Chancen gegenüber den Risiken abzuwägen. Es ist zu hoffen, dass sich ein Umdenken auch für die Indikationsstellung der Strahlentherapie durchsetzt. So hat eine aktuelle Studie an 1184 Frauen ergeben, dass die nach brusterhaltender Operation notwendige Strahlentherapie bei Patientinnen im Frühstadium durch eine „akzelerierte“ Teilbrustbestrahlung auf fünf Tage verkürzt werden kann, ohne dass dies 5 Jahre danach einen Unterschied im Gesamtüberleben oder in der Rezidivrate gehabt hat [30].

\section{"Choosing Wisely"-Initiative}

Es wäre wünschenswert, wenn die 2011 in den USA gestartete „Choosing Wisely“Initiative auch hier in Deutschland und möglichst auch von der Onkologie aufgegriffen würde. „Choosing Wisely“ verfolgt das Ziel, die offene Diskussion zwischen der Ärzteschaft, den Patienten und der Öffentlichkeit zum Thema Überversorgung zu fördern. Kern von Choosing Wisely sind Top-5-Listen aus jeder klinischen Fachdisziplin, die fünf medizinische Maß- 
nahmen enthalten, bei der gegenwärtig eine Überversorgung festzustellen ist und deshalb ein verstärkter Bedarf an Information und „Shared Decision Making“ besteht.

Die Ausgangsthese von „Shared Decision Making“ (partizipative oder gemeinsame Entscheidungsfindung) ist, dass es nicht nur eine aus Expertensicht zu bestimmende optimale Therapie für den einzelnen Patienten gibt, sondern dass die aktive Mitarbeit der Patienten und die Einbeziehung der persönlichen, körperlichen und seelischen Gegebenheiten notwendig ist, um die optimale Therapie in einer gemeinsamen Entscheidung festzulegen [1]. Im neuen Patientenrechtegesetz, das seit 2013 gültig ist, ist dies ausdrücklich verankert. So soll der Arzt Werte und Präferenz des Patienten bezüglich Aufklärung, Diagnose und Therapie eruieren und berücksichtigen.

Leider wird die gemeinsame Entscheidungsfindung im Klinikalltag häufig gleichgesetzt bzw. verwechselt mit dem Konstrukt des „informed consent“ (Informierte Entscheidung): Der Schwerpunkt liegt hier auf Information. Der Patient wird umfassend aufgeklärt und stimmt der vorgeschlagenen Therapieoption zu (Tumorkonferenz, Leitlinien).

\section{Patientenaufklärung}

Ganz wichtig ist, dass Leitlinien bzw. Empfehlungen der jeweiligen Fachgesellschaften keine rechtliche Verpflichtung beinhalten. Vorausgesetzt, der Patient wird über mögliche Risiken aufgeklärt, darf der behandelnde Arzt im Interesse des Patienten und der Weiterentwicklung der medizinischen Wissenschaft von den Leitlinien abweichen und neue bzw. unkonventionelle Behandlungsmethoden einsetzen. Denn rechtlich gesehen ist die Basis der heilberuflichen Profession die sogenannte Therapiefreiheit. Das bedeutet, dass der Behandler darüber entscheiden kann, ob und wie im konkreten Einzelfall diagnostisch oder therapeutisch vorzugehen ist.

Für den Patienten bedeutet dies aber auch, dass auf der Grundlage des verfassungsrechtlich garantierten Selbstbestimmungsrechtes, welches das Recht auf freie Arztwahl und auch das Recht auf eine (vermeintlich) medizinisch unvernünftige Entscheidung beinhaltet, jede in den Leitlinien fixierte Therapie abgelehnt werden darf. Weiterführende Informationen $\mathrm{zu}$ diesem wichtigen Thema erhalten Sie bei der Gesellschaft für Biologische Krebsabwehr e.V. (GfBK-Info Patientenaufklärung und die GfBK-Info Selbstbestimmung und Therapiefreiheit).

\section{Fazit}

Wir brauchen in der heutigen Medizin eine neue Kultur der Integration! Neu im Sinne davon, dass die Medizin wieder als Dienstleistung am Patienten verstanden wird. Dies kann jedoch nur gelingen, wenn alle Fachdisziplinen die gemeinsame Entscheidungsfindung mit dem Patienten pflegen und wenn auch die Patienten akzeptiert und weiterbetreut werden, die eine leitliniengerechte Medizin ablehnen.

Eine am Patienten orientierte ,individualisierte“ ganzheitliche Beratung erfordert mehr Zeit, lohnt sich aber, da die Patienten besser in der Lage sind, die für sie stimmige Entscheidung und deren Konsequenzen mitzutragen. Entscheidend für mich ist wiederum das Zitat von Prof. Michael Untsch: „Wenn die Angst der Hauptgrund für die Indikation ist, bleiben sachliche Gründe oft auf der Strecke.“ Ich hoffe, dass viele Onkologen umdenken und sich gemeinsam mit den Patienten die Zeit nehmen können, die die Patienten brauchen, damit nicht aus Angst, sondern aus Zuversicht eine individuelle Entscheidung getroffen werden kann.

Autorenerklärung: Die Autorin erklärt, dass keine finanziellen Interessenkonflikte im Zusammenhang mit diesem Beitrag bestehen.

online:

http://dx.doi.org/10.1055/s-0042-108141

\section{Korrespondenzadresse}

Dr. med. Nicole Weis

Gesellschaft für Biologische Krebsabwehr e.V. Voßstr. 3

69115 Heidelberg

E-Mail: n.weis@biokrebs.de

\section{Literatur}

[1] Badura B, Hart D, Schellschmidt H. Bürgerorientierung des Gesundheitswesens. Selbstbestimmung, Schutz, Beteiligung. Gutachten im Auftrag des Ministeriums für Arbeit, Gesundheit und Soziales des Landes Nordrhein-Westfalen. Mit Beiträgen von Bernhard Badura, Robert Francke, Winfried Göpfert, Dieter Hart, Ellis Huber, Germanus Hungeling, Christoph Kranich, Henner Schellschmidt und Franz Stuchlik. BadenBaden: Nomos; 1999: 16

[2] Ballard-Barbash R, Friedenreich CM, Courneya KS et al. Physical activity, biomarkers, and disease outcomes in cancer survivors: A systematic review. J Natl Cancer Inst 2012; 104(11): 815-40

[3] Bijker N, Meijnen P, Peterse JL et al. Breastconserving treatment with or without radiotherapy in ductal carcinoma-in-situ: Tenyear results of European Organisation for Research and Treatment of Cancer randomized phase III Trial 10853-A study by the EORTC Breast Cancer Cooperative Group and EORTC Radiotherapy Group. J Clin Oncol 2006; 24: 3381-7

[4] Bradshaw PT, Ibrahim JG, Khankari N et al. Post-diagnosis physical activity and survival after breast cancer diagnosis: the Long Island Breast Cancer Study. Breast Cancer Res Treat 2014; 145(3): 735-42

[5] Cold S, Düring M, Ewertz $M$ et al. Does timing of adjuvant chemotherapy influence the prognosis after early breast cancer? Results of the Danish Breast Cancer Cooperative Group (DBCG). Br J Cancer 2005; 93(6): 627-32

[6] Cuzick J, Sestak I, Pinder SE et al. Effect of tamoxifen and radiotherapy in women with locally excised ductal carcinoma in situ: long-term results from the UK/ANZ-DCIStrial. Lancet Oncol 2011; 12: 21-9

[7] Davies C, Pan H, Godwin J, Gray R, Arriagada R, Raina V, Abraham M, Alencar VHM, Badran A, Bonfill X et al, for the Adjuvant Tamoxifen Longer Against Shorter (ATLAS) Collaborative Group. Long-term effects of continuing adjuvant tamoxifen to 10 years versus stopping at 5 years after diagnosis of oestrogen receptor-positive breast cancer: ATLAS, a randomised trial. The Lancet 2013; 381(9869): 805-16

[8] Deber RB, Kraetschmer N, Urowitz S, Sharpe $\mathrm{N}$. Do people want to be autonomous patients? Preferred roles in treatment decisionmaking in several patient populations. Health Expect 2007; 10: 248-58

[9] EBCTCG (Early Breast Cancer Trialists' Collaborative Group). Effect of radiotherapy after mastectomy and axillary surgery on 10year recurrence and 20-year breast cancer mortality: meta-analysis of individual patient data for 8135 women in 22 randomised trials. The Lancet 2014; 383: 2127-35 
[10] Ellis P, Barrett-Lee P, Johnson L et al. Sequential docetaxel as adjuvant chemotherapy for early breast cancer (TACT): an openlabel, phase III, randomised controlled trial. Lancet 2009; 373(9676): 1681-92

[11] Giesen A et al. Körperliche Aktivität und Sport bei Mammakarzinom - ein Update. Dtsch Z Onkol 2015; 47: 100-4

[12] Giuliano AE, Hunt KK, Ballman KV et al. Axillary dissection vs no axillary dissection in women with invasive breast cancer and sentinel node metastasis: a randomized clinical trial. JAMA 2011; 305(6): 569-75

[13] Hall E et al. Comparison of patient reported quality of life and impact of treatment side effects experienced with a taxane-containing regimen and standard anthracycline based chemotherapy for early breast cancer: 6 year results from the UK TACT trial (CRUK/ 01/001). Eur J Cancer 2014; 50(14): 237589

[14] https://idw-online.de/de/news639718

[15] Keisch M, Vicini F, Beitsch P et al. American Society of Breast Surgeons MammoSite Radiation Therapy System Registry Trial: ductal carcinoma-in-situ subset analysis-4-year data in 194 treated lesions. Am J Surg 2009; 198(4): 505-7

[16] Keller-Matschke K. Und wo bleibt der Patient? Dtsch Z Onkol 2012; 44: 178-9

[17] Klein F. Den Patienten als Partner sehen. Im Focus Onkologie 2015; 18(1-2): 12-5

[18] Kunkler I et al. The PRIME 2 trial: Avoiding radiotherapy is an option for some older patients with breast cancer. S2-01. 36th San Antonio Breast Cancer Symposium 10.-14.12.2013, San Antonio, SA

[19] Lattrich Claus. Therapie von Mammakarzinomen. TumorDiagn u Ther 2010; 31: 324-7

[20] Lehmann C, Koch U, Mehnert A. Die Bedeutung der Arzt-Patient-Kommunikation für die psychische Belastung und die Inanspruchnahme von Unterstützungsangeboten bei Krebspatienten: Ein Literaturüberblick über den gegenwärtigen Forschungsstand unter besonderer Berücksichtigung patientenseitiger Präferenzen. Psychother Psych Med 2009; 59: e3-e27

[21] Little P, Everitt H, Williamson I et al. Observational study of effect of patient centredness and positive approach on outcomes of general practice consultations. BM] 2001; 323(7318): 908-11

\section{Summary}

Expectations and reality: Patient competence and guideline-oriented medicine a plea for humane and integrative medicine

Guidelines from expert organizations are often the basis of therapy decisions. However, in some cases, „guideline recommendations“ are not regarded as „recommendations“ but as part and parcel of undisputed medical and thus indispensable therapy catalogue. Such an approach is usually a recipe for conflict between the medical practitioner and the patient. This article serves as a plea for a humane and integrative medicine that uses breast cancer therapy as an example. In this context, the patient and the doctor jointly make a decision, even when in certain cases this implies that the guideline recommendations are rejected based on individual considerations.

Keywords: Patients competence, guidelines, breast cancer, oncological therapy, antihormons, chemotherapy, radiation therapy, lymphonodectomy

[22] Matthiessen PF. Leitlinien in der Onkologie Pro \& Contra. Dtsch ZOnkol 2004; 36: 135-7

[23] Möbus V. In: Kreienberg R et al, eds. Mammakarzinom interdisziplinär. Berlin, Heidelberg: Springer; 2010: 181, Tab. 16.2, und 277-96

[24] Neuman J, Korenstein D, Ross JS, Keyhani S. Prevalence of financial conflicts of interest among panel members producing clinical practice guidelines in Canada and United States: cross sectional study. BMJ 2011; 343: d5621

[25] Neumaier J, Untch M. Taxan bei frühem Brustkrebs: Mehr Schaden als Nutzen? ImFocus Onkologie 2015; 18(3): 32-3. DOI: 10.1007/s15015-015-1651-2

[26] Palta M, Palta P, Bhavsar NA et al. The use of adjuvant radiotherapy in elderly patients with early-stage breast cancer: Changes in practice patterns after publication of Cancer and Leukemia Group B 9343. Cancer 2015; 121(2): 188-93

[27] Platsch K-D. Die Medizin heilen. München: Systemische Medizin; 2014: 65

[28] Roberts CS el al. Influence of physician communication on newly diagnosed breast cancer patients psychologic adjustment and decision-making. Cancer 1994; 74(1 Suppl): 336-41

[29] Rudolph I et al. Der Informationsbedarf von Patienten. Dtsch Med Wochenschr 2015; 140: e43-e47
[30] Strnad V, Ott O], Hildebrandt G, KauerDorner $D$, Knauerhase $H$, on behalf of the Groupe Européen de Curiethérapie of European Society for Radiotherapy and Oncology (GEC-ESTRO) et al. 5-year results of accelerated partial breast irradiation using sole interstitial multicatheter brachytherapy versus whole-breast irradiation with boost after breast-conserving surgery for low-risk invasive and in-situ carcinoma of the female breast: a randomised, phase 3, non-inferiority trial. The Lancet 2016; 387: 229-38

[31] Vodermaier A. Prophylaktische Chirurgie bei Brust- und Eierstockkrebsrisiko aus psychologischer Perspektive. Dissertation. München: LMU München, Fakultät für Psychologie und Pädagogik; 2005

[32] Vodermaier A, Caspari C, Köhm J, Bauerfeind I, Kahlert S, Untch M. Shared decision-making in primary breast cancer. Z Ärztl Fortbild Qualitatssich 2004 Mar; 98(2):127-33

[33] Wärnberg F et al. Effect of Radiotherapy after breast-conserving surgery for ductal carcinoma in situ: 20 years follow-up in the randomized SweDICS Trial. J Clin Oncol 2014; 32(32): 3613-8

[34] Williams PT. Significantly greater reduction in breast cancer mortality from post-diagnosis running than walking. Int J Cancer 2014; 135(5): 1195-202 